\title{
Patterns and Trends in Diagnostic Tests Used for Detection of Colorectal Cancer after Screening with the Immunochemical Fecal Occult Blood Test in Japan
}

\author{
Junta Yamamichi', Kahori Seto ${ }^{1 *}$, Shiro Hinotsu' ${ }^{2}$, Koichi Nagata ${ }^{3,4}$, Yasutoshi Kobayashi', \\ Hisashi Urushihara ${ }^{5}$, Koji Kawakami1" \\ ${ }^{1}$ Department of Pharmacoepidemiology, Graduate School of Medicine and Public Health, Kyoto University, \\ Kyoto, Japan \\ ${ }^{2}$ Center for Innovative Clinical Medicine, Okayama University Hospital, Okayama, Japan \\ ${ }^{3}$ Cancer Screening Technology Division, Research Center for Cancer Prevention and Screening, National Cancer \\ Center, Tokyo, Japan \\ ${ }^{4}$ Department of Radiology, Jichi Medical University, Tochigi, Japan \\ ${ }^{5}$ Division of Drug Development and Regulatory Science, Faculty of Pharmacy, Keio University, Tokyo, Japan \\ Email: "kawakami.koji.4e@kyoto-u.ac.jp
}

Received 22 May 2015; accepted 23 August 2015; published 26 August 2015

Copyright (C) 2015 by authors and Scientific Research Publishing Inc.

This work is licensed under the Creative Commons Attribution International License (CC BY). http://creativecommons.org/licenses/by/4.0/

(c) (i) Open Access

\section{Abstract}

According to the guidelines by the Japanese government, optical colonoscopy is the most strongly recommended diagnostic test after screening with the immunochemical fecal occult blood test (iFOBT), followed by double-contrast barium enema (BE) or sigmoidoscopy. Our study was to assess patterns and trends of colorectal cancer (CRC) diagnostic testing within 2 years after iFOBT. We analyzed both iFOBT results and claims data provided by employee health insurance societies in Japan from 2005 to 2010 . 25,596 enrollees underwent iFOBT screening. The positive rate was $5.1 \%$. 32.3\% of those positive underwent diagnostic tests and $1.0 \%$ (12 patients) were confirmed as having cancer. The most common test was optical colonoscopy $(77.2 \%$ of total tests), followed by BE (16.2\%). From 2006 to 2009 , the rate of optical colonoscopy for females increased from $55 \%$ to $82 \%$ and that of $\mathrm{BE}$ declined from $36 \%$ to $12 \%$, while no significant changes were seen for males. Only one-third of those who tested positive underwent diagnostic test in the 2 years following screening iFOBT. As official guidelines for diagnostic testing of CRC recommend, optical colonoscopy is now the most commonly used diagnostic test after positive iFOBT result for enrollees in employee health insurance societies in Japan.

\footnotetext{
${ }^{*}$ Co-first author.

"Corresponding author.
}

How to cite this paper: Yamamichi, J., Seto, K., Hinotsu, S., Nagata, K., Kobayashi, Y., Urushihara, H. and Kawakami, K. (2015) Patterns and Trends in Diagnostic Tests Used for Detection of Colorectal Cancer after Screening with the Immunochemical Fecal Occult Blood Test in Japan. Open Journal of Clinical Diagnostics, 5, 107-116. http://dx.doi.org/10.4236/ojcd.2015.53018 
Keywords

Colorectal Cancer Screening, Diagnostic Test, iFOBT, Optical Colonoscopy, Barium Enema

\section{Introduction}

Colorectal cancer (CRC) represents the third-highest cause of mortality among males and the highest among females in Japan [1], with an age-standardized mortality rate of 11.8 per 100,000 persons [2]. This mortality rate is equal to or worse than those of the US and UK [2]. CRC screening is a practical way of reducing mortality due to CRC [3]. However, the screening rate in Japan was low at 24.9\% [4]. The Japanese government implemented the Cancer Control Act in 2007 to promote the early detection of cancer by targeting a screening rate of $>50 \%$ in the general population within 5 years. In the US, about $60 \%$ of targeted Americans have any CRC screening test [5].

In Japan, fecal occult blood testing (FOBT) is recommended for annual screening from age 40 years using the two-consecutive sample fecal immunochemical test method based on scientific evaluation by the Research Task Force on Cancer Screening Programs at the Ministry of Health, Labour, and Welfare (MHLW) in 2005. On the other hand, the US multi-society consensus guideline recommends screening by FOBT every year, sigmoidoscopy every 5 years combined with FOBT every 3 years, or (optical) colonoscopy every 10 years for adults aged 50 to 75 years [6]. In the UK, the NHS Bowel Cancer Screening Programme offers FOBT every 2 years for those aged 60 to 74 years [7]. The Japanese government's 2007 guideline further recommends optical colonoscopy as the first-choice diagnostic test when the result of screening immunochemical FOBT (iFOBT) is positive, with double-contrast barium enema (BE) or sigmoidoscopy being the second options. iFOBT-based screening programs in Japan are managed independently by local governments or employee health insurance societies. As a consequence, a variety of screening practices are observed across the country [8], but no specific quantification of patterns and trends in CRC testing among employee health insurers in Japan has yet appeared, despite the fact that such an insurance covers one-quarter of the population.

The present study aimed to evaluate patterns of diagnostic test use after iFOBT among employee health insurers and trends in CRC testing in Japan from 2006 to 2009. It also assessed compliance with diagnostic test recommendations by the MHLW.

\section{Materials and Methods}

\subsection{Study Population and Data Set}

The national universal health care system regulates all reimbursements of health care expenses in Japan by the single fee schedule [9]. Payment is reimbursed by employee health insurance societies or national health insurance program according to the enrollment. Copies of insurance claims data from April 2005 to May 2010 were obtained from three employee health insurance societies. These health insurance societies also provided us with annual voluntary screening iFOBT results of their enrollees from April 2005 to May 2010. Both claims data and iFOBT results were combined for the analyses. The claims data consisted of three types of claim relating to inpatient $(n=3445)$, outpatient $(n=264,193)$ and pharmacy benefits $(n=127,068)$. Since enrollees underwent multiple iFOBTs, these results were grouped by the first and second checkup rounds for our study purpose. The first checkup round was the first iFOBT the enrollee underwent during the study period, and the second checkup round was the iFOBT undergone by those enrollees whose result in the first checkup round was negative.

\subsection{CRC Diagnostic Tests and Treatments}

All enrollees were followed for patterns of clinical procedures undergone within 2 years after iFOBT or until their next checkup, whichever came first, regardless of the test results. All records were examined for the time period from screening iFOBT to treatment, if any. Treatments for CRC include polypectomy, surgery, and chemotherapy. Since patients visited healthcare providers for various reasons, data were limited to claims with the following diseases or symptoms related to CRC using specified ICD-10 codes: for malignant diseases, C18-C20; and for benign diseases, D12, D37.4, D37.5, K62, K63, K92, R19.5, and R77.8. In cases of multiple diseases or 
symptoms in a claim, the most severe one was chosen to represent the particular patient; this is because healthcare providers file an insurance claim only once per month by consolidating all services provided to each patient within that month. Diseases or symptoms under clinical suspicion were excluded from the analyses because they were not confirmed. When multiple procedures such as computed tomography (CT) were repeatedly provided in consecutive months, they were counted as a single procedure. Chemotherapies for CRC were identified when the dispensed drug was coded as L01 or L02 in the Anatomical Therapeutic Chemical Classification [10].

\subsection{Analyses}

Patterns of CRC diagnostic test use and treatments were analyzed separately according to the test results at each checkup round. To depict the trend of test use, the use rates for optical colonoscopy, BE, and other tests that are not recommended by the guidelines were calculated as a percentage of all tests within each year. Here, optical colonoscopy included colonoscopy, sigmoidoscopy, rectoscopy, and anoscopy, albeit that sigmoidoscopy, rectoscopy, and anoscopy were infrequent. Other tests included conventional CT, magnetic resonance imaging (MRI), et cetera. In accordance with the national fee schedule, concurrent endoscopic testing with polypectomy was only charged as polypectomy, and thus these endoscopic tests could not be counted separately from polypectomy.

The study plan was approved by Kyoto University Graduate School and Faculty of Medicine, Ethics Committeein January 2011.

95\% confidence intervals (CIs) for percentages were calculated assuming an underlying normal distribution. Hypotheses were tested by two sample t-test assuming equal variances at $\alpha=0.05$ level of significance (twosided). Statistical analyses were performed using R version 2.14.2 (The R Foundation for Statistical Computing, Vienna, Austria).

\section{Results}

\subsection{Flow of CRC Screening and Characteristics of Study Population}

Figure 1 shows the flow of all enrollees analyzed. At the first checkup round, 25,596 enrollees were screened, of whom 1,304 (5.1\%; 95\% CI, 4.8 - 5.4) had a positive result and the remaining 24,292 (94.9\%; 95\% CI, 94.6 - 95.2) had a negative result. $32.3 \%$ of those who tested positive at the first checkup round underwent diagnostic tests, of whom $31.1 \%$ were treated by polypectomy, surgery, or chemotherapy. A proportion (53.5\%) of those who had a negative result at the first checkup round went to the second checkup

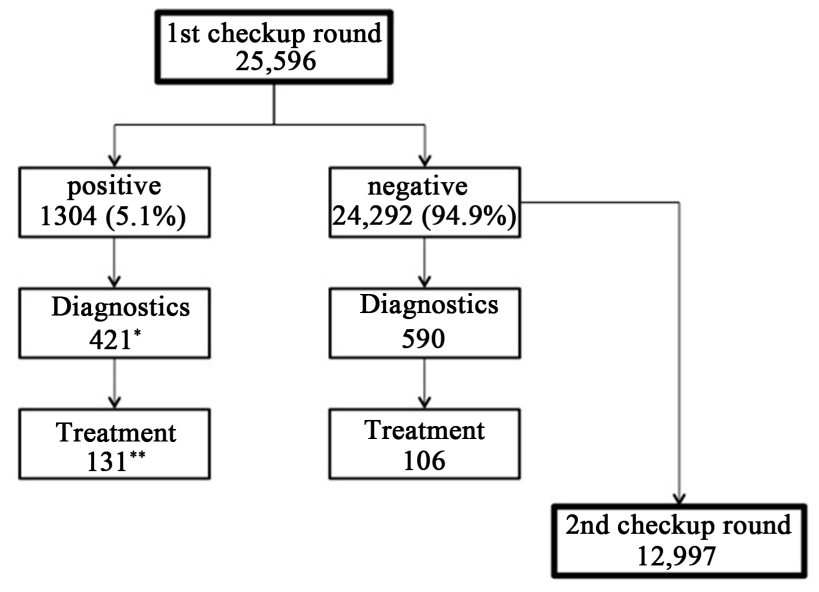

(a)

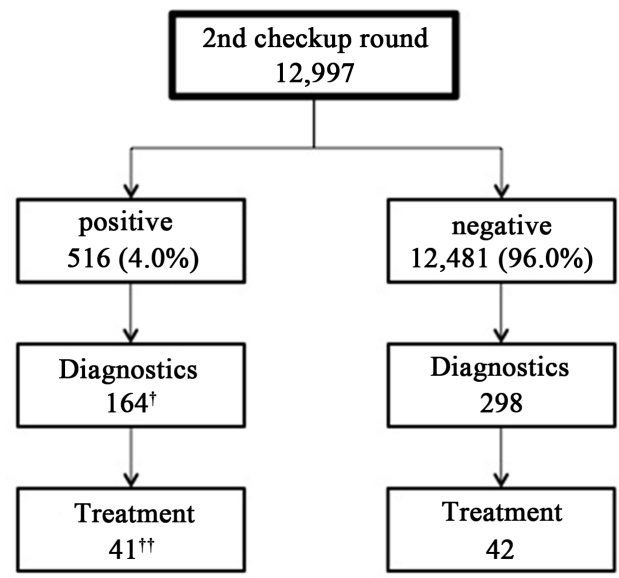

(b)

Figure 1. Flow of subjects within 2 years after the first (a) and second checkup round (b). ${ }^{*} 32.3 \%$ of those who tested positive at the first checkup round underwent diagnostic tests. ${ }^{* *} 10.0 \%$ of those who tested positive at the first checkup round were treated; $31.1 \%$ of those who underwent diagnostic tests were treated by polypectomy, surgery, or chemotherapy. ${ }^{\dagger} 31.8 \%$ of those who tested positive at the second checkup round underwent diagnostic tests. ${ }^{+{ }^{\dagger}} 7.9 \%$ of those who tested positive at the second checkup round were treated; $25.0 \%$ of those who underwent diagnostic tests were treated by polypectomy, surgery, or chemotherapy. 
( $n=12,997)$. At the second checkup round, 516 (4.0\%; 95\% CI, $3.7-4.3$ ) had a positive result. 31.8\% of those who tested positive at the second checkup round underwent diagnostic tests, of whom $25.0 \%$ were treated. Table 1 shows the demographics of these two rounds. $62 \%$ of those tested in each checkup round were male. The study population was relatively young since they were employees (72.6\%) and their dependents. Those with a positive result tended to be male (71.3\%) and older (mean age: 49.1) than those with a negative result.

\subsection{Patterns of Diagnostic Procedures}

Table 2 describes the patterns of procedures for those with a positive result at the first checkup round. All screenes were followed until the initial treatment started. The patterns are separately shown as a flow categorized by the recommended guidelines for CRC diagnosis in Japan. Both polypectomy and biopsy are independently included in Table 2 because optical colonoscopy may have been required before such procedures but were not distinguishable by claims data. Endoscopic tests other than optical colonoscopy were also included $(n=$ 7). The percentage of patients who underwent diagnostic tests was highest for optical colonoscopy (24.9\%; 95\% CI, 22.6 - 27.2), followed by BE (5.2\%; 95\% CI, 4.0 - 6.4) and other tests that are not recommended (2.1\%; 95\% CI, 1.3 - 2.9). Optical colonoscopy accounted for $77.2 \%$ of all tests, while BE accounted for $16.2 \%$. The combination of biopsy and polypectomy after optical colonoscopy was complex, but on average, about half of colonoscopic tests were followed by biopsy (41.5\%). In the BE group, $42.6 \%$ of patients further underwent optical colonoscopy. The use rate of optical colonoscopy among those who underwent other tests that are not recommended by the guidelines was even higher (71.4\%). Nine patients with cancer were identified in the optical colonoscopy group, one in the BE group, and two in the other tests group based on disease codes in a claim. Closely similar characteristics were observed in the analysis of the second checkup round as well (Table 3).

\subsection{Influence of Gender and Age on Test Use}

Diagnostic test use differed by gender and age. Male had a lower rate of undergoing diagnostic tests after positive iFOBT result than female (29.4\% and 34.8\%, respectively, in the first checkup round). Among those with a

Table 1. Demographics of the study population.

\begin{tabular}{|c|c|c|c|c|}
\hline & First positive & First negative & Second positive & Second negative \\
\hline Subjects, n (\%) & 1304 (5) & 24,292 (95) & $516(4)$ & $12,481(96)$ \\
\hline \multicolumn{5}{|l|}{ Sex, n (\%) } \\
\hline Male & $930(71)$ & $14,952(62)$ & 353 (68) & $7641(61)$ \\
\hline Female & 374 (29) & $9340(38)$ & $163(32)$ & 4840 (39) \\
\hline Mean agea, y & 49.1 & 45.9 & 49.5 & 47.1 \\
\hline IQR Agea, y & $40-59$ & $37-55$ & $40-59$ & $39-55$ \\
\hline \multicolumn{5}{|l|}{ Age category ${ }^{\mathrm{a}}, \mathrm{n}(\%)$} \\
\hline $20-29 y$ & $11(0.8)$ & 447 (1.8) & $1(0.2)$ & $56(0.4)$ \\
\hline $30-39 y$ & 300 (23) & $7623(31)$ & $106(21)$ & 3416 (27) \\
\hline $40-49 y$ & 315 (24) & $6805(28)$ & $143(28)$ & 3875 (31) \\
\hline $50-59 y$ & 395 (30) & $6247(26)$ & $152(30)$ & 3513 (28) \\
\hline $60-69 y$ & 248 (19) & $2894(12)$ & $107(21)$ & $1514(12)$ \\
\hline $70-75 y$ & $16(1.2)$ & $132(0.5)$ & $5(1.0)$ & $75(0.6)$ \\
\hline Employee $^{\mathrm{b}}$, n (\%) & $1049(80)$ & $17,526(72)$ & $404(78)$ & 8914 (71) \\
\hline
\end{tabular}

${ }^{\mathrm{a}}$ Age was computed as on December 31st of each study year. ${ }^{\mathrm{b}}$ Non-employees consisted of the dependents of employees. They were covered by the same employee health insurances. 
Table 2. Patterns of clinical procedures for those with a positive iFOBT result at the first checkup round.

\begin{tabular}{|c|c|c|}
\hline Pattern of clinical procedures & Patient & Confirmed Cancer \\
\hline Primarily recommended diagnostic test: colonoscopy, $n\left(\%{ }^{\mathrm{a}}\right)$ & $325(24.9)$ & 9 \\
\hline iFOBT(+) -> colonoscopy & $242(18.6)$ & \\
\hline ->biopsy & 85 & \\
\hline ->biopsy->polypectomy & 21 & \\
\hline ->biopsy->surgery & 1 & 1 \\
\hline ->polypectomy & 10 & \\
\hline iFOBT(+) -> biopsy & $11(0.8)$ & \\
\hline ->polypectomy & 2 & 1 \\
\hline ->polypectomy->surgery->chemotherapy & 1 & 1 \\
\hline ->surgery-> chemotherapy & 1 & 1 \\
\hline ->chemotherapy & 1 & 1 \\
\hline iFOBT(+) ->polypectomy & $72(5.5)$ & 3 \\
\hline ->chemotherapy & 1 & 1 \\
\hline Secondarily recommended diagnostic test: $\mathrm{BE}, \mathbf{n}\left(\%^{\mathrm{a}}\right)$ & $68(5.2)$ & 1 \\
\hline $\mathrm{iFOBT}(+)->\mathrm{BE}$ & $62(4.8)$ & \\
\hline ->colonoscopy & 17 & \\
\hline ->colonoscopy->biopsy & 6 & \\
\hline ->colonoscopy->biopsy->polypectomy & 1 & \\
\hline ->colonoscopy->polypectomy & 2 & \\
\hline ->biopsy & 2 & \\
\hline ->polypectomy & 8 & \\
\hline iFOBT(+) -> BE, CT & $6(0.5)$ & \\
\hline ->biopsy ->polypectomy & 1 & \\
\hline ->polypectomy & 1 & 1 \\
\hline Other tests that are not recommended by the guidelines, $n\left(\%{ }^{\mathrm{a}}\right)$ & $28(2.1)$ & 2 \\
\hline iFOBT(+) -> CT & $20(1.5)$ & \\
\hline ->colonoscopy & 11 & \\
\hline ->colonoscopy->biopsy & 5 & \\
\hline ->colonoscopy->biopsy->surgery -> chemotherapy & 1 & 1 \\
\hline ->biopsy & 2 & \\
\hline ->polypectomy & 3 & \\
\hline iFOBT(+) -> MRI ->polypectomy & $1(0.1)$ & \\
\hline iFOBT(+) -> CT, MRI & $4(0.3)$ & \\
\hline ->polypectomy & 3 & \\
\hline iFOBT(+) -> FOBT & $2(0.2)$ & \\
\hline iFOBT(+) -> surgery ->chemotherapy & $1(0.1)$ & 1 \\
\hline
\end{tabular}

${ }^{\mathrm{a}}$ Percentage of the total number of iFOBT $(+)$. According to the national fee schedule, biopsy was a removal for a pathologic diagnostic test of a piece of tissue and polypectomy was an endoscopic removal of a whole polyp as a treatment. 
Table 3. Patterns of clinical procedures for those with a positive iFOBT result at the second checkup round.

\begin{tabular}{|c|c|c|}
\hline Pattern of clinical procedures & Patients & Confirmed Cancer \\
\hline Primarily recommended diagnostic test: colonoscopy, $n\left(\%{ }^{\mathrm{a}}\right)$ & $118(22.9)$ & 5 \\
\hline iFOBT(+) -> colonoscopy & $93(18.0)$ & \\
\hline ->biopsy & 30 & \\
\hline ->biopsy->polypectomy & 8 & 2 \\
\hline ->polypectomy & 4 & \\
\hline ->polypectomy->surgery & 1 & 1 \\
\hline iFOBT(+) -> biopsy & $7(1.4)$ & \\
\hline ->polypectomy & 2 & \\
\hline iFOBT(+) ->polypectomy & $18(3.5)$ & 2 \\
\hline Secondarily recommended diagnostic test: $\mathrm{BE}, \mathrm{n}\left(\%^{\mathrm{a}}\right)$ & $35(6.8)$ & 2 \\
\hline $\mathrm{iFOBT}(+)->\mathrm{BE}$ & $32(6.2)$ & \\
\hline ->colonoscopy & 5 & \\
\hline ->colonoscopy->biopsy & 3 & \\
\hline ->colonoscopy->polypectomy & 1 & \\
\hline ->biopsy & 1 & \\
\hline ->polypectomy & 3 & 1 \\
\hline $\mathrm{iFOBT}(+)->\mathrm{BE}, \mathrm{CT}$ & $3(0.6)$ & \\
\hline ->biopsy->polypectomy->surgery & 1 & 1 \\
\hline ->polypectomy & 1 & \\
\hline Other tests that are not recommended by the guidelines, $n\left(\%{ }^{\mathrm{a}}\right)$ & $11(2.1)$ & 1 \\
\hline iFOBT(+) -> CT & $7(1.4)$ & \\
\hline ->colonoscopy & 5 & \\
\hline ->colonoscopy->biopsy & 2 & \\
\hline ->colonoscopy->biopsy->surgery -> chemotherapy & 1 & 1 \\
\hline ->polypectomy & 1 & \\
\hline iFOBT(+) -> MRI -> colonoscopy ->biopsy & $1(0.2)$ & \\
\hline iFOBT(+) -> FOBT & $3(0.6)$ & \\
\hline ->colonoscopy->polypectomy & 1 & \\
\hline
\end{tabular}

${ }^{\mathrm{a}}$ Percentage of the total number of iFOBT (2nd, +).

positive result in the first checkup round, males composed $67 \%$ uses of optical colonoscopy, $45 \%$ of BE, and $65 \%$ of CT. With regard to age, the mean age of patients was 49.6 years for optical colonoscopy, 46.0 years for $\mathrm{BE}$, and 54.2 years for CT. A similar trend was observed for those who tested positive at the second checkup round (51.1, 49.6, and 53.4 years, respectively).

\subsection{Cancer Prevalence}

Classification of cancer and benign disease among treated patients was estimated using disease codes (Table 4). Here, any diseases or symptoms in claims were excluded if they were under clinical suspicion and not confirmed. Computing the ratios of the incidences of cancer estimated by the 2-year follow-up of claims, patients with a positive iFOBT test can be thought of as having at least a 33- to 40 -fold higher risk of cancer in this population than patients with a negative test result. 
Table 4. Disease classification among treated patients estimated by claims data.

\begin{tabular}{ccccc}
\hline & First positive & First negative & Second positive & Second negative \\
\hline Total subjects, n (\%) & $1304(100)$ & $24,292(100)$ & $516(100)$ & $12,481(100)$ \\
Cancer $^{\mathrm{a}}$, n (\%) & $12(1.0)$ & $8(0.03)$ & $8(1.6)$ & $5(0.04)$ \\
Benign disease $^{\mathrm{b}}, \mathrm{n}(\%)$ & $89(6.8)$ & $83(0.3)$ & $34(6.6)$ & $35(0.3)$ \\
\hline
\end{tabular}

${ }^{\mathrm{a} I C D}-10$ codes C18-C20. ${ }^{\mathrm{b}} \mathrm{ICD}-10$ codes D12, D37.4, D37.5, K62, K63, K92, R19.5, R77.8.

\subsection{Trends in Test Use}

Changes in the utilization of diagnostic tests were analyzed from 2006 to 2009, using data from patients who had a positive test result in the first checkup round. Due to the small number of tests underwent by male in 2006, trend analysis for male were limited from 2007 to 2009. The trend in utilization differed between males and females. Males showed no significant change over time (Figure 2(a)): about $80 \%$ of the diagnostic tests were optical colonoscopy, with both BE and others accounting for about $10 \%$ of all tests. On the contrary, females showed an apparent change in utilization (Figure 2(b)), with the use of optical colonoscopy increasing from 55\% to $82 \%$ of all tests and that of BE declining from $36 \%$ to $12 \%$. The difference between males and females in test utilization was diminishing. A slight difference in mean elapsed time from a positive test result to diagnostic optical colonoscopy were seen at 2.9 months for males and 3.8 months for females $(p=0.05)$.

\section{Discussion}

The important findings of this study were the low rate of diagnostic test use after a positive iFOBT result and low adherence to annual screening. Only one-third of those who tested positive received a diagnostic test and a half of screenees missed opportunities of annual screening. 59\% of patients were reported to receive follow-up testing in the Veterans Health Administration in the US [11]. As an issue of Japanese CRC screening program, the large variability in adherence rates to diagnostic follow-up examinations after positive iFOBT was discussed in the previous study [12]. To maximize the benefit of the screening program, a more systematic effort to involve more screenees in the diagnostic tests may be warranted. For instance, incomplete recommendations by physicians or health insurers for follow-up diagnostic tests could have a major impact on the low rate of undergoing diagnostic tests and a poor compliance with annual screening [13]. Good physician-patient relationship helps better understanding of the importance of screening program and more appropriate participation by patients [14].

When analyzing patterns and trends in the use of diagnostic tests, optical colonoscopy was the most common diagnostic test for those with a positive iFOBT result, followed by BE. As suggested in previous studies of the advanced features of optical colonoscopy in cancer diagnosis [15] [16], ongoing clinical improvements in methodology could lead to a substantial expansion of its use. The use of optical colonoscopy by males prevailed against others. On the contrary, in earlier years, females tended to use tests other than optical colonoscopy more frequently than males, but the rate of optical colonoscopy approximated that for males in 2009. A temporal increase in optical colonoscopy use and decrease in BE was apparently observed also in U.S. Medicare enrollees from 1998 to 2005 [17]. In particular, those tested by BE tended to be young or female. With respect to such gender differences, a higher use of screening endoscopy by male was reported in the U.S. National Health Interview Surveys as well [18]. As others have mentioned about increasing use of optical colonoscopy [18] [19], we consider that the relatively high cost and anticipated capacity limitations in the ability to provide optical colonoscopy should also be taken into account under efforts to ensure equal access to care and avoid leaving patients unscreened or undiagnosed.

A notable aspect of the study population was that one-third of it was under 40 years old, despite fact that, in Japan, it is recommended that people at average risk should be screened by iFOBT every year after the age of 40 . This is because employee health insurance societies freely decided the age at which enrollees are screened. A peculiar feature of the Japanese system is that the screening program is managed independently by the respective employee health insurance society and is even financed by the same insurance society. This age distribution could have affected the use of diagnostic tests due to differences in the attitude toward cancer prevention between generations. The quality of screening program like CRC screening might be affected by factors like ap- 


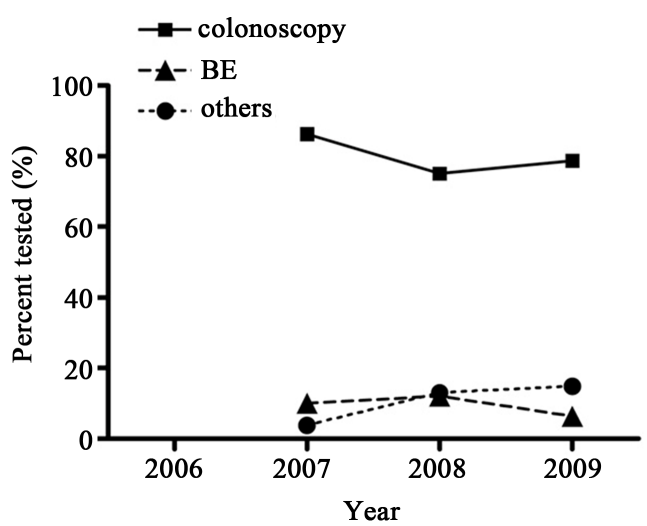

(a)

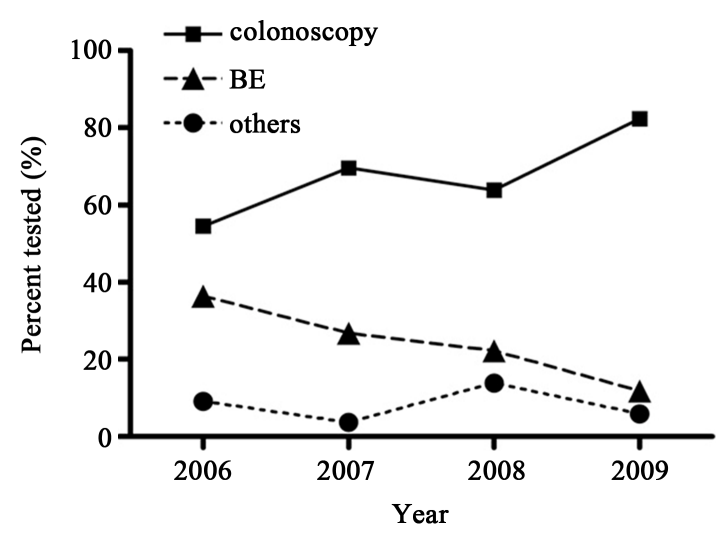

(b)

Figure 2. Time trends in the utilization of diagnostic tests. (a) Males from 2007 to 2009; (b) Females from 2006 to 2009. Rates were calculated as a percentage of overall tests undergone in each year from those who tested positive at the first checkup round. Males underwent 130, 100, and 47 tests overall in each year from 2007 to 2009 , respectively. Females underwent 22, 56, 36, and 17 tests overall in each year from 2006 to 2009, respectively.

propriateness in selection of targeted population. The cost effectiveness of such a voluntary CRC screening program requires further consideration, particularly given such wide coverage in age for iFOBT screening and small proportion of patients receiving follow-up diagnostic tests and treatment.

The prevalence of cancer detected in the present study was 4.5 per 10,000 individuals. This rate is comparable to that reported by the Japanese Society of Gastroenterological Cancer Screening in 2006. Although our study design and claims data set including many younger subjects could not support a valid comparison of test performance with other studies, it is true that iFOBT apparently failed to detect some CRC cases (8 out of 20). Thus, there might be an allowable scope for activities to increase the performance. The quality control in conducting test is important to maximize the benefit of screening programs from a population perspective. For example, the test performance might be influenced by the difference in the number of fecal samples and the method for sample collection. Further study is necessary to discuss the performance improvement.

Among those who received diagnostic tests, compliance with the guidelines for CRC diagnostic tests was quite good, with 10 of 12 cancers (83.3\%) diagnosed following the guideline. Physicians in Japan now appear to apply the recommendations. An important aspect of adherence to CRC testing in this population is found as the low rate of diagnostic testing (32.3\%). We cannot compare the numbers equivalently, but $47.2 \%$ of US Medicare enrollees were estimated to receive tests as recommended by the guidelines [20] in 2005 [17]. The US guidelines practically include more types of CRC tests as voluntary opportunistic screening than the Japanese guidelines. Further, the 2010 National Health Interview Survey of the US general population indicated that 58.3\% of those aged 50 to 75 years met the recommendations for CRC testing [3]. In Italy, only 11.2\% of colon cancer patients who accessed an Italian Local Health Agency in 2007 were found to have undergone the sequence of tests recommended by the official guidelines (colonoscopy and closed biopsy) [21]. The authors noted that this low compliance in Italy was probably due to the long waiting times and long waiting lists for colonoscopy in the public healthcare system.

Although weaknesses of our study may include a retrospective database study design and relatively young study population, our data set was large and standardized across nation to generalize the results to other screening programs in Japan. However, there were other limitations. First, not all enrollees visited healthcare providers to see doctors even if they had CRC-related symptoms during our study period. In addition, some might not have seen doctors due to asymptomatic CRC or might have withdrawn from their health insurance within the 2-year follow-up period. Second, since the present analyses were based on claims data, medical records were not directly checked for the complete diagnostic opinion such as a type of polyps and a stage of CRC, and a degree of misclassification of diseases may have been accordingly present. Third, people were free to take any screening test at their own cost. Although these are thought to be few in number, it was not possible to investigate such patterns of testing using this data set since the tests completed without submitting a claim were not included. Finally, each health insurer had its own policy for the conduct of screening programs and the follow-up of those with a positive result. 


\section{Conclusion}

To depict the patterns and trends in CRC diagnostic test use in the employee health insurance, societies those in total cover about one-quarter of the Japanese population and thus play an important role in controlling cancer mortality in Japan, the present study analyzed the claims data from a subset of employee health insurance societies. These societies differ in their attitude to cancer screening programs, with some providing free screening while others not encouraging screening. Therefore, we think that policymakers could promote and bolster such screening programs to achieve targeted screening rates at a more rapid pace with stronger financial and institutional support to the insurance societies. Importantly, we unveiled the low rate of diagnostic test use after a positive iFOBT result and poor adherence to annual screening. Thus, additional systematic efforts toward intense follow-up of those testing positive in screening and improved adherence to annual screening should be considered. Lastly, optical colonoscopy is now the most commonly used diagnostic test for both male and female enrollees of employee health insurance societies in Japan. The study results suggest that healthcare providers in Japan generally follow the MHLW's official guidelines for diagnostic testing of CRC, with the use of BE declining substantially.

\section{Acknowledgements}

The authors thank Japan Medical Data Center Co., Ltd. (JMDC), Tokyo, Japan. Copies of the insurance claims and screening iFOBT results were collected and collated by JMDC and analyzed under a research collaboration agreement between JMDC and Kyoto University.

\section{Disclosure of Potential Conflicts of Interest}

J. Y. is an employee of Canon Inc.; K. K. is a scientific advisor to Olympus Corporation. No research funds were received from any companies for this research.

\section{References}

[1] Matsuda, T., Marugame, T., Kamo, K., Katanoda, K., Ajiki, W. and Sobue, T. (2012) Cancer Incidence and Incidence Rates in Japan in 2006: Based on Data from 15 Population-Based Cancer Registries in the Monitoring of Cancer Incidence in Japan (MCIJ) Project. Japanese Journal of Clinical Oncology, 42, 139-147. http://dx.doi.org/10.1093/jjco/hyr184

[2] Ferlay, J., Shin, H.R., Bray, F., Forman, D., Mathers, C. and Parkin, D.M. (2010) GLOBOCAN 2008 v2.0, Cancer Incidence and Mortality Worldwide: IARC CancerBase No. 10. International Agency for Research on Cancer. http://globocan.iarc.fr

[3] Pignone, M.P., Rich, M., Teutsch, S., Berg, A. and Lohr, K. Screening for Colorectal Cancer in Adults, Agency for Healthcare Research and Quality (U.S.). Report No. 02-S003. http://www.ncbi.nlm.nih.gov/books/NBK42629/

[4] Ministry of Health, Labour and Welfare (Japan) (2007) Comprehensive Survey of Living Conditions. http://www.mhlw.go.jp/english/database/db-hss/cslc-index.html

[5] Shapiro, J.A., Klabunde, C.N., Thompson, T.D., Nadel, M.R., Seeff. L.C. and White, A. (2012) Patterns of Colorectal Cancer Test Use, including CT Colonography, in the 2010 National Health Interview Survey. Cancer Epidemiology, Biomarkers \& Prevention, 21, 895-904. http://dx.doi.org/10.1158/1055-9965.EPI-12-0192

[6] Levin, B., Lieberman, D.A., McFarland, B., Smith, R.A., Brooks, D., Andrews, K.S., Dash, C., Giardiello, F.M., Glick, S., Levin, T.R., Pickhardt, P., Rex, D.K., Thorson, A. and Winawer, S.J. (2008) Screening and Surveillance for the Early Detection of Colorectal Cancer and Adenomatous Polyps, 2008: A Joint Guideline from the American Cancer Society, the US Multi-Society Task Force on Colorectal Cancer, and the American College of Radiology. CA: A Cancer Journal for Clinicians, 58, 130-160. http://dx.doi.org/10.3322/CA.2007.0018

[7] The NHS Bowel Cancer Screening Programme http://www.cancerscreening.nhs.uk/bowel/

[8] Imamura, A. (2009) Current Status of Colorectal Cancer Screening in Japan. Gastrointestinal Endoscopy, 51, 26752689.

[9] Ikegami, N. and Campbell, J.C. (1995) Medical Care in Japan. New England Journal of Medicine, 333, $1295-1299$. http://dx.doi.org/10.1056/NEJM199511093331922

[10] European Pharmaceutical Market Research Association (2012) The Anatomical Classification of Pharmaceutical Products. http://www.ephmra.org/classification/anatomical-classification.aspx

[11] Etzioni, D.A., Yano, E.M., Rubenstein, L.V., Lee, M.L., Ko, C.Y., Brook, R.H., Parkerton, P.H. and Asch, S.M. (2006) 
Measuring the Quality of Colorectal Cancer Screening: The Importance of Follow-Up. Diseases of the Colon \& Rectum, 49, 1002-1010. http://dx.doi.org/10.1007/s10350-006-0533-2

[12] Saito, H. (2006) Colorectal Cancer Screening Using Immunochemical Faecal Occult Blood Testing in Japan. Journal of Medical Screening, 13, S6-S7.

[13] Jimbo, M., Myers, R.E., Meyer, B., Hyslop, T., Cocroft, J., Turner, B.J. and Weinberg, D.S. (2007) Reasons Patients with a Positive Fecal Occult Blood Test Result Do Not Undergo Complete Diagnostic Evaluation. The Annals of Family Medicine, 7, 11-16. http://dx.doi.org/10.1370/afm.906

[14] Hassan, C., Laghi, A., Zullo, A., Iafrate, F. and Morini, S. (2008) Q\&A on Diagnosis, Screening and Follow-Up of Colorectal Neoplasia. Digestive and Liver Disease, 40, 85-96. http://dx.doi.org/10.1016/j.dld.2007.09.007

[15] Kobayashi, Y., Kudo, S.E, Miyachi, H., Hosoya, T., Ikehara, N., Ohtsuka, K., Kashida, H., Hamatani, S., Hinotsu, S. and Kawakami, K. (2011) Clinical Usefulness of Pit Patterns for Detecting Colonic Lesions Requiring Surgical Treatment. International Journal of Colorectal Disease, 26, 1531-1540. http://dx.doi.org/10.1007/s00384-011-1246-0

[16] Kobayashi, Y., Hayashino, Y., Takagaki, N., Hinotsu, S., Jackson, J.L. and Kawakami, K. (2012) Diagnostic Performance of Chromoendoscopy and Narrow Band Imaging for Colonic Neoplasms: Meta-Analysis. Colorectal Disease, 14, 18-28. http://dx.doi.org/10.1111/j.1463-1318.2010.02449.x

[17] Schenck, A.P., Peacock, S.C., Klabunde, C.N., Lapin, P., Coan, J.F. and Brown, M.L. (2009) Trends in Colorectal Cancer Test Use in the Medicare Population, 1998-2005. American Journal of Preventive Medicine, 37, 1-7. http://dx.doi.org/10.1016/j.amepre.2009.03.009

[18] Meissner, H.I., Breen, N., Klabunde, C.N. and Vernon, S.W. (2006) Patterns of Colorectal Cancer Screening Uptake among Men and Women in the United States. Cancer Epidemiology, Biomarkers \& Prevention, 15, 389-394. http://dx.doi.org/10.1158/1055-9965.EPI-05-0678

[19] Fenton, J.J., Cai, Y., Green, P., Beckett, L.A., Franks, P. and Baldwin, L.M. (2008) Trends in Colorectal Cancer Testing among Medicare Subpopulations. American Journal of Preventive Medicine, 35, 194-202. http://dx.doi.org/10.1016/j.amepre.2008.05.029

[20] Centers for Medicare and Medicaid Services. (2004) National Coverage Determination (NCD) for Colorectal Cancer Screening Tests (210.3).

http://www.cms.gov/medicare-coverage-database/details/ncd-details.aspx?NCDId=281\&ncdver=1\&CALId=97\&ver=7 \&CalName=Prothrombin+Time+and+Fecal+Occult+Blood+(Revision+of+ICD-9-CM+Codes+for+Injury+to+Gastroin testinal+Tract)\&bc=AAQAAAAABAAA\&.

[21] Antonelli, D. and Petrigni, C. (2011) Investigation into the Actual Application of the Diagnostic and Therapeutic Guidelines for Colon Cancer. Italian Journal of Public Health, 8, 310-317. 